\title{
Protocadherin-10 acts as a tumor suppressor gene, and is frequently downregulated by promoter methylation in pancreatic cancer cells
}

\author{
CHAN QIU, XIAONA BU and ZHENG JIANG
}

Department of Gastroenterology, First Affiliated Hospital, Chongqing Medical University, Chongqing 400016, P.R. China

Received December 28, 2015; Accepted February 11, 2016

DOI: $10.3892 /$ or.2016.4793

\begin{abstract}
Protocadherin-10 (PCDH10), a member of nonclustered protocadherin family which plays important roles in calcium-dependent cell-cell signal transduction and adhesion. PCDH10 functions as a tumor suppressor gene and its expression is downregulated by promoter methylation in many malignances. In the present study, we explored PCDH10 expression and promoter methylation status, and its biological effects in pancreatic cancer cells, and furthermore, we explored the mechanism of PCDH10 preliminary in pancreatic cancer cells. The mRNA level of PCDH10 was detected by semi-quantitative reverse transcription PCR and promoter methylation status examined by methylationspecific PCR in the pancreatic cancer cells (Capan-1, Panc-1, AsPC-1 and BxPC-3) as well as the human normal pancreatic ductal epithelial cells HPDE6-C7 which was used as a control. The human pancreatic cells were transfected with plasmid pcDNA3.1-PCDH10 or pcDNA3.1 by Lipofectamine 2000. The biological function of PCDH10 in pancreatic cancer cells was determined by CCK-8 assay, colony formation assay, flow cytometry, Transwell invasion assay and woundhealing assay. The levels of apoptosis related proteins were examined by western blotting. PCDH10 expression was obviously downregulated in the pancreatic cancer cells (Capan-1, Panc-1, BxPC-3) compared to the normal pancreatic ductal epithelial cells. PCDH10 promoter methylation was observed in the two pancreatic cancer cells Capan-1 and BxPC-3, and the expression of $\mathrm{PCDH} 10$ could be restored after treating with 5-aza-2'-deoxycytidine and trichostatin A in the two cell types. Overexpression of PCDH10 can inhibit the proliferation, migration, invasion ability of pancreatic cancer cells and induce apoptosis. Ectopic expression of PCDH10
\end{abstract}

Correspondence to: Dr Zheng Jiang, Department of Gastroenterology, First Affiliated Hospital, Chongqing Medical University, Chongqing 400016, P.R.China

E-mail: jiangz1753@163.com

Key words: protocadherin-10, methylation, pancreatic cancer, tumor suppressor gene could increase the levels of PARP, caspase-3, caspase-9 and decrease the level of bcl-2, AKT and p-AKT. PCDH10 acts as a tumor suppressor gene, and is frequently down-regulated by promoter methylation in pancreatic cancer cells. PCDH10 may induce cancer cell apoptosis via the AKT pathway.

\section{Introduction}

Pancreatic cancer is a common malignant tumor of the digestive system. Most pancreatic cancers are pancreatic ductal adenocarcinomas. Pancreatic cancer is among the deadliest cancer in the world, its 5-year survival rate is only $4 \%$ and after surgical radical resection is less than $20 \%$ (1). The poor survival of pancreatic cancer is due to its late diagnosis, early metastasis, and insufficient therapy. Unfortunately, the survival rate of patients with pancreatic cancer have not been increased in recent years. Deeper understanding of pancreatic cancer based on the molecular mechanisms, has revealed that pancreatic cancer is due to genetic and epigenetic alterations (2). New tumor makers which could help detect pancreatic cancer in the early stage and provide potential therapeutic targets are necessary and valuable.

Epigenetics modification is defined as the modification influencing gene transcription and translation inherited without changing the DNA sequence, including DNA methylation, histone modifications, chromatin remodeling and RNA interference (3). DNA methylation is a common epigenetic modification reported to affect chromatin structure and gene stability $(4,5)$. The aberrant DNA promoter methylation of tumor suppressor genes (TSGs) play an important role in mostly cancer including esophageal, gastric and colorectal cancer. In addition, several TSGs including p16, UCHL1 and ROR2 have been confirmed with promoter aberrant methylation resulting in silence (6-8).

Protocadherin-10 (PCDH10) belongs to the protocadherin family, locating in human chromosome 4q28.3. Protocadherins are predominantly expressed in the nervous system, playing an important role in signaling pathways and cell-cell adhesion $(9,10)$. Accumulating evidence indicates that protocadherins including PCDH8, PCDH17 and PCDH20 can suppress tumorigenesis and tumor progress in multiple carcinomas (11-13). Early studies have demonstrated that PCDH10 is frequently downregulated by promoter 
Table I. RT-PCR and MSP primers.

\begin{tabular}{|c|c|c|c|c|c|}
\hline PCR & Primer & Sequence (5'-3') & $\begin{array}{l}\text { Product } \\
\text { size (bp) }\end{array}$ & $\begin{array}{l}\text { PCR } \\
\text { cycles }\end{array}$ & $\begin{array}{c}\text { Annealing } \\
\text { temperature }\left({ }^{\circ} \mathrm{C}\right)\end{array}$ \\
\hline \multirow[t]{4}{*}{ RT-PCR } & PCDH10-sense & ACTGCTATCAGGTATGCCTG & 219 & 32 & 55 \\
\hline & PCDH10-antisense & GTCTGTCAACTAGATAGCTG & & & \\
\hline & $\beta$-actin-sense & CTCCATCCTGGCCTCGCTGT & 268 & 23 & 55 \\
\hline & $\beta$-actin-antisense & GCTGTCACCTTCACCGTTCC & & & \\
\hline \multirow[t]{4}{*}{ MSP } & PCDH10-bm1 & TCGTTAAATAGATACGTTACGC & 153 & 40 & 60 \\
\hline & PCDH10-bm2 & TAAAAACTAAAAACTTTCCGCG & & & \\
\hline & PCDH10-bu1 & GTTGTTAAATAGATATGTTATGT & 155 & 40 & 58 \\
\hline & PCDH10-bu2 & СТАААААСТАААААСТТТССАСА & & & \\
\hline
\end{tabular}

methylation and functions as a tumor suppressor gene in gastric, colorectal cancer, lung cancer and in many other carcinomas (14-16). However, the research on PCDH10 in pancreatic cancer is rare, its expression, methylation status and biological function in pancreatic cancer are not know yet. We examined PCDH10 mRNA expression and promoter methylation status in pancreatic cancer cells, and the overexpression of PCDH10 to detect its biological function in pancreatic cancer cells. In addition, we preliminarily explored the mechanism of PCDH10 in pancreatic cancer cells.

\section{Materials and methods}

Cell lines. Human pancreatic cancer cell lines (Capan-1, Panc-1, AsPC-1 and BxPC-3) and human normal pancreatic ductal epithelial cell line (HPDE6-C7) were from Chongqing Key Laboratory of Molecular Oncology and Epigenetics. The cells were maintained at $37^{\circ} \mathrm{C}$ in Dulbecco's modified Eagle's medium (DMEM) or RPMI-1640 medium (HyClone Laboratories, Inc., Logan, UT, USA) with $10 \%$ fetal bovine serum (FBS; Gibco, Grand Island, NY, USA).

Semi-quantitative reverse transcription PCR (RT-PCR) analysis. Total RNA was isolated from cells by TRIzol (Invitrogen), RNA concentration and purity were tested, the value of $\mathrm{OD}(260) / \mathrm{OD}(280)$ should be at the range of 1.8-2.0. Total RNA was reverse transcribed into cDNA according to the reverse transcription kit (Promega, Madison, WI, USA). RT-PCR amplification primer synthesis by Takara Bio (Shiga, Japan), the RT-PCR primer sequences are listed in Table I. RT-PCR steps according to the GoTaq polymerase (Promega) instructions, and the amplification conditions were: $95^{\circ} \mathrm{C}$ for $2 \mathrm{~min} ; 95^{\circ} \mathrm{C}$ for $30 \mathrm{sec}, 55^{\circ} \mathrm{C}$ for $30 \mathrm{sec}, 70^{\circ} \mathrm{C}$ for $30 \mathrm{sec}$ (PCDH10 using 32 cycles, $\beta$-actin using 23 cycles); and $70^{\circ} \mathrm{C}$ for $3 \mathrm{~min}$. PCR products were analyzed on $2 \%$ agarose gel electrophoresis, and Bio-Rad gel imaging system was used for analysis of exposure and quantity.

DNA bisulfite treatment and methylation-specific PCR $(M S P)$ assays. Genomic DNA was extracted from cells with using DNA Extraction kits (Tiangen, Co., Ltd., Beijing, China), DNA bisulfite modification and methylation status of PCDH10 in pancreatic cancer cells and normal pancreatic ductal epithelial cells were detected by using the EZ DNA Methylation-Gold ${ }^{\mathrm{TM}}$ kit; Zymo Research Corp., Irvine, CA, USA). The methylated and unmethylated MSP primers are shown in Table I (17). Methylation specific PCR (MSP) was performed using AmpliTaq Gold (Applied Biosystems). PCR products were analyzed on $2 \%$ agarose gel electrophoresis.

5-aza-2'-deoxycytidine (Aza) and trichostain A (TSA) treatment. Following overnight incubation, Capan-1 and BxPC-3 cells were treated with 5-aza-2'-deoxycytidine (SigmaAldrich; $10 \mu \mathrm{M})$ for 3 days, and then trichostain A (100 ng/ ml; Cayman Chemical, Ann Arbor, MI, USA) was added to cell medium. After $24 \mathrm{~h}$, the total RNA and DNA were extracted.

Transfection and $G 418$ selection. The cells (Capan-1 and BxPC-3) were plated in 6-well plates, transfection was performed at $75 \%$ confluence. Discarding the medium, the cells were washed with serum-free medium 2 times, then each well was added with $1 \mathrm{ml}$ serum-free medium. A total of $4 \mu \mathrm{g}$ plasmid (pcDNA3.1-PCDH10 or pcDNA3.1-vector)

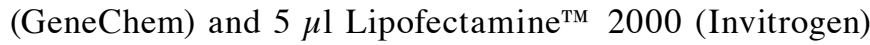
were diluted by $500 \mu 1$ serum-free medium, respectively, and allowed to incubate for $5 \mathrm{~min}$ at room temperature. Plasmid and Lipofectamine ${ }^{\mathrm{TM}} 2000$ diluent were mixed and incubated for $20 \mathrm{~min}$ at room temperature, and each well was added with $1 \mathrm{ml}$ the above mixture. After culturing for $4-6 \mathrm{~h}$ at $37^{\circ} \mathrm{C}$, the medium was discarded adding $2 \mathrm{ml}$ medium with $10 \%$ FBS. After 48 h, G418 (Amresco, Solon, OH, USA) was added into the medium (G418 concentration was tested before, Capan-1 $800 \mu \mathrm{g} / \mathrm{ml}$ and BxPC-3 $700 \mu \mathrm{g} / \mathrm{ml}$ ) for $\sim 2$ weeks until single clones formed, then maintaining the concentration of G418 by half the culture continued. The medium containing G418 was changed each 3 days.

The expression of PCDH1O in transfected cells. Cells (Capan-1 and BxPC-3) were transfected with plasmid pcDNA3.1-PCDH10 or pcDNA3.1-vector, after $48 \mathrm{~h}$, the total RNA and proteins were extracted. RT-PCR was used to examine the expression of PCDH10 in cells at mRNA level. Western blot analysis was performed to test the protein level: total proteins were extracted with PIPA lysate buffer 
(Beyotime Institute of Biotechnology, Shanghai, China), and concentration was measured with BCA kit (Beyotime Institute of Biotechnology). After polyacrylamide gel electrophoresis, proteins were transfered onto polyvinylidene fluoride (PVDF) membranes (Beyotime Institute of Biotechnology). The membranes were left in $5 \%$ skimmed milk for $2 \mathrm{~h}$ at room temperature, then incubated with primary antibodies overnight [PCDH10 mouse anti-human monoclonal antibody (Abnova, Taipei, Taiwan); $\beta$-actin mouse anti-human monoclonal antibody (Cell Signaling Technology, Inc., Danvers, MA, USA)] at $4^{\circ} \mathrm{C}$, washed with TBST buffer, incubated with the second antibodies (goat anti-mouse; Cell Signaling Technology) with horseradish peroxidase (HRP) for $2 \mathrm{~h}$ at room temperature. The membranes were washed again, then the proteins were visualized with BeyoECL Plus kits (Beyotime Institute of Biotechnology). Results were analyzed with fusion software.

Cell proliferation colony formation assays. The cells (Capan-1 and BxPC-3) were seeded in a 96-well plate at a density of $5 \times 10^{3}$ cells/well, each sample set in three wells and allowed to culture overnight. Cells were transfected with plasmid pcDNA3.1-PCDH10 or pcDNA3.1-vector Cell Counting kit-8 (Dojindo Molecular Technologies, Inc., Rockville, MD, USA) was used to to evaluated cells proliferation ability, the detection points were 24,48 and $72 \mathrm{~h}$ after transfection.

The cells (Capan-1 and BxPC-3) stably transfected with pcDNA3.1-vector or pcDNA3.1-PCDH10 were seeded in a 6 -well plate at a density of $1 \times 10^{3}$ cells/well. Each sample set in three wells. For two weeks, the clones ( $\geq 50$ cells) were counted after fixing with paraformaldehyde and staining with Gentian violet.

Wound-healing and Transwell invasion assays. Cell (BxPC-3) migration was assessed with scratch wound-healing assay. Stably transfected cells were seeded in a 6-well plate until confluence, cell layer was scratched with a $2 \mu$ l pipette tip and washed with PBS, maintained in serum-free DMEM. Cells were photographed under a phase-contrast microscope after incubation for 0,24 and $48 \mathrm{~h}$.

The invasion ability of cells (BxPC-3) was measured by 24-well Transwell ${ }^{\mathrm{TM}}$ (Corning, Inc., Corning, NY, USA) with Matrigel (BD Biosciences, San Diego, CA, USA). The Matrigel was diluted with serum-free medium according to the instructions, added into the upper chamber, incubated at $37^{\circ} \mathrm{C}$ until the Matrigel solidified. A total of $700 \mu \mathrm{l}$ medium containing $10 \%$ FBS was added into the lower chamber, $100 \mu \mathrm{l}$ serum-free medium with $2 \times 10^{4}$ cells was added to the upper chamber. After incubation for $48 \mathrm{~h}$, cells that migrated were fixed and stained, then counted under a microscope in three fields.

Cell apoptosis assays. The apoptosis of Capan-1 cells was detected by combining flow cytometry and Annexin V-FITC/PI staining. After transfection for $48 \mathrm{~h}$, the cells were digested with EDTA-free trypsin enzyme and collected, washed with PBS once gently and collected again. Cells were resuspended with $1 \mathrm{ml}$

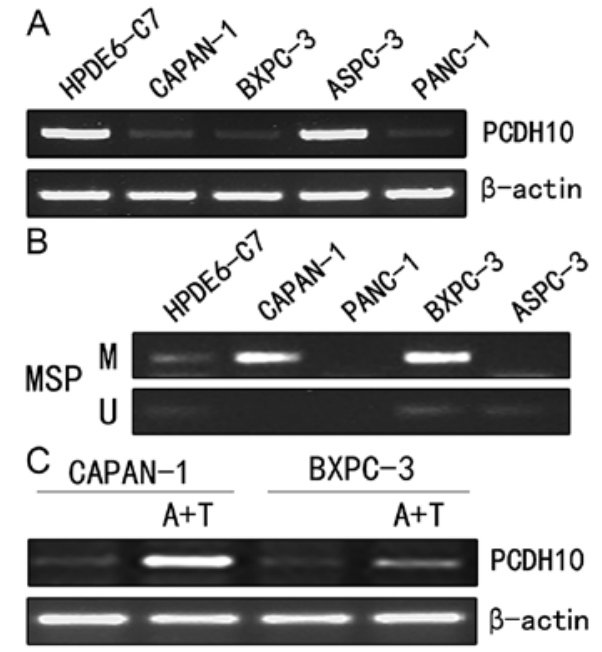

Figure 1. PCDH10 expression is downregulated and correlated with promoter methylation in pancreatic cancers. (A) The expression of PCDH10 in pancreatic cancer cells and normal cells examined by RT-PCR. (B) The promoter methylation status of PCDH10 in pancreatic cancer cells and normal cells tested by MSP. (C) PCDH10 mRNA expression after treating with Aza and TSA detected by RT-PCR. M, methylation; U, unmethylation; Aza, 5-aza2'-deoxycytidine; TSA, trichostain A.

PBS, counted and transfered to $1.5 \mathrm{ml}$ EP tube. Taking $5-10 \times 10^{4}$ cells, 1,000 g/5 min, discarding the clear liquid, $195 \mu \mathrm{l}$ Annexin V-FITC was added to suspend the cells, subsequently adding $5 \mu \mathrm{l}$ Annexin V-FITC. Adding $10 \mu \mathrm{l}$ organism iodide dyeing liquid, incubated for 10-20 min at room temperature, then, evaluated by flow cytometry.

Expression of the key cell apoptosis regulators examined by western blot analysis. After polyacrylamide gel electrophoresis, proteins were transfered onto PVDF membranes. The membranes were left in 5\% skimmed milk for $2 \mathrm{~h}$ at room temperature then incubated with primary antibodies overnight (AKT, p-AKT, PARP, caspase-3, cytochrome-9 and Bcl-2 are rabbit anti-human polyclonal antibodies; Cell Signaling Technology), at $4^{\circ} \mathrm{C}$, washed with TBST buffer, incubated in the second antibody (goat anti-rabbit; Cell Signaling Technology) with HRP mark, for $2 \mathrm{~h}$ at room temperature. The membranes were washed again, then the proteins were visualized with BeyoECL Plus kits. Data were analyzed with fusion software.

Statistical analysis. Experiments were performed in triplicate. Data are presented as the mean \pm SD and analyzed with Student's t-test, and the analyses were performed with the SPSS version 21 . $\mathrm{P}<0.05$ was considered to indicate a statistically significant result.

\section{Results}

PCDH10 is downregulated in pancreatic cancer cells. We used RT-PCR to examine the expression of PCDH10 in pancreatic cancer cells and the normal pancreatic ductal epithelial cells. The result demonstrated that PCDH10 was downregulated in pancreatic cancer cells (Capan-1, Panc-1 and BxPC-3) compared to the normal pancreatic ductal epithelial cells (Fig. 1A). 


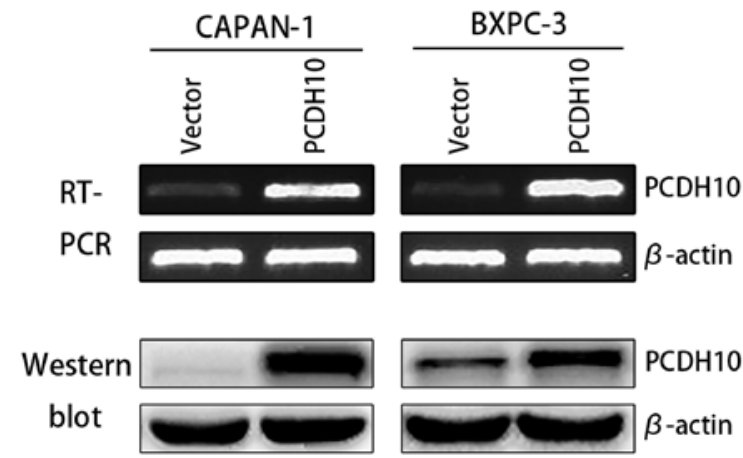

Figure 2. The expression of PCDH10 was significantly increased in cells transfected with PCDH10 examined by RT-PCR and western blot analysis. Vector, cells transfected with pcDNA3.1-vector; PCDH10, cells transfected with pcDNA3.1-PCDH10.
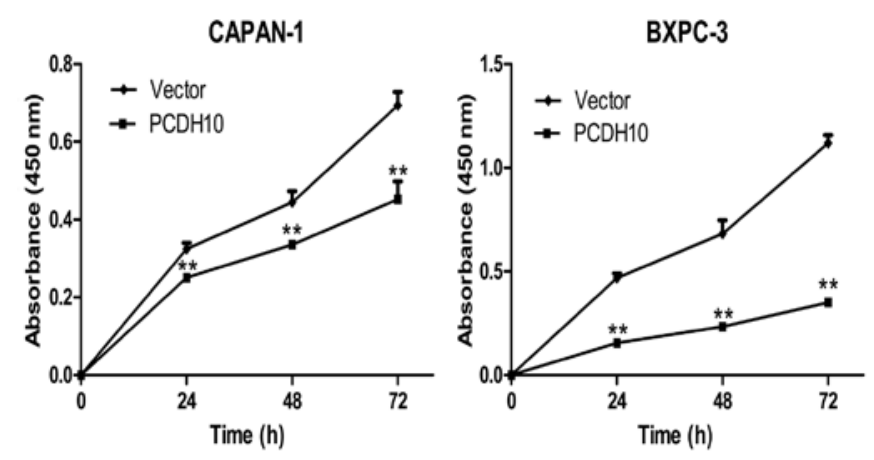

Figure 3. The effects of PCDH10 overexpression on cell proliferation ${ }^{* *} \mathrm{P}<0.01$ compared to the vector.

PCDH10 is frequently downregulated with promoter methylation in pancreatic cancer cells and restored by methylation inhibitor histone deacetylase inhibitor. MSP was performed to analyze the methylation status in the $\mathrm{CpG}$ island of PCDH10 promoter in pancreatic cancer cells as well as the normal pancreatic ductal epithelial cells. The cells (Capan-1 and BxPC-3) in which PCDH10 was silenced displayed promoter methylation, while was not found in normal cells (Fig. 1B). Then, the cells were treated with methylation inhibitor Aza and histone deacetylase inhibitor TSA. As expected, the expression of PCDH10 was recovered (Fig. 1C). The results revealed that DNA promoter methylation contributes to $\mathrm{PCDH} 10$ silence in pancreatic cancer cells.

The expression of PCDH1O significantly increased after transfection. RT-PCR results showed that the expression of PCDH10 mRNA in cells transfected with pcDNA3.1-PCDH10 was significantly higher than those in cells transfected with pcDNA3.1-vector. Western blot analysis showed that the expression of PCDH10 protein in cells transfected with PCDH10 was increased compared with cells transfected with plasmid vector $(\mathrm{P}<0.01 ;$ Fig. 2$)$.

Re-expression of PCDH1O inhibits the proliferation and colony formation of pancreatic cancer cells. Several studies have found that PCDH10 can inhibit cancer growth, whether this applies to pancreatic cancer is unclear. To ascertain this question, cell proliferation and colony formation assays were
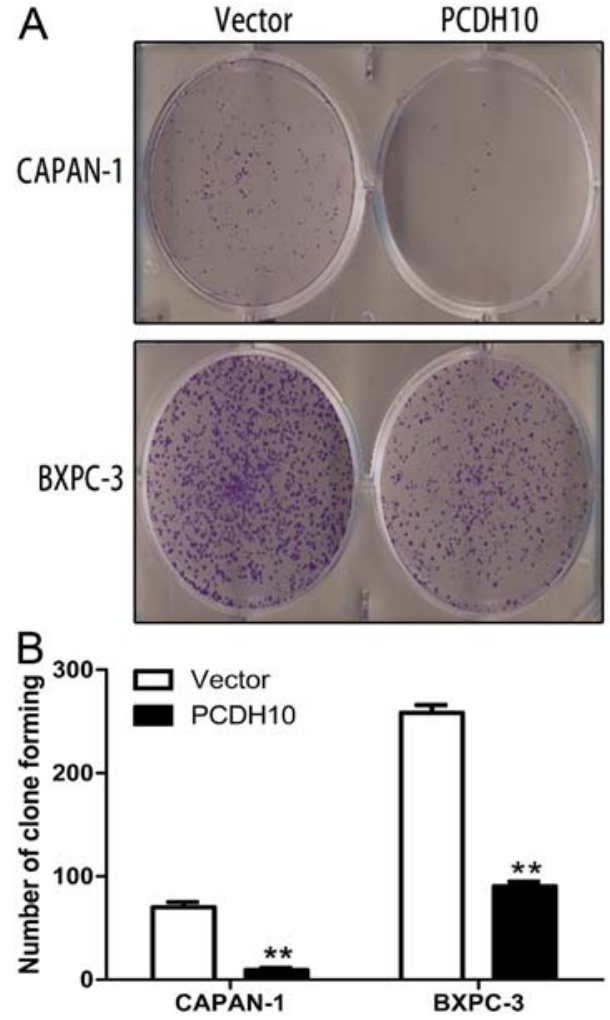

Figure 4. The effects of PCDH10 overexpression on cell colony forming. (A) Representative images of colony formation. (B) The quantitative analysis of colony formation. ${ }^{* *} \mathrm{P}<0.01$ compared to the vector (crystal violet staining).

performed in the study. Cell Counting kit- 8 assay demonstrated that the cells transfected with PCDH10 grew significant slower than cells transfected with vector at 24,48 and $72 \mathrm{~h}(\mathrm{P}<0.01$; Fig. 3). Furthermore, the colony formation assay showed the PCDH10-transfected cells formed markedly fewer and smaller colonies than the vector-transfected cells $(\mathrm{P}<0.01$; Fig. 4). These results revel that $\mathrm{PCDH} 10$ can inhibit pancreatic cancer cell growth.

PCDH1O inhibits the migration and invasion ability of pancreatic cancer cells. Wound-healing and Transwell ${ }^{\mathrm{TM}}$ invasion assays were performed to test the effect of PCDH10 on the migration and invasion ability of pancreatic cancer cells BxPC-3. Cells transfected with PCDH10 spread significantly more slowly than cells transfected with vector in the woundhealing test $\left(\mathrm{P}<0.01\right.$; Fig. 5). The Transwell ${ }^{\mathrm{TM}}$ invasion assay showed a marked suppression of invasion in the PCDH10transfected cells $(\mathrm{P}<0.01 ;$ Fig. 6$)$. The above results suggested that PCDH10 may play an important role in metastatic diffusion of pancreatic cancer cells.

Ectopic PCDH10 expression induces apoptosis of pancreatic cancer cells. We performed flow cytometry combined with Annexin V-FITC/PI staining to explore the effect of PCDH10 on apoptosis in Capan-1. Apoptosis of cells transfected with PCDH10 was obviously increased compared with the cells transfected with vector $(\mathrm{P}<0.05$; Fig. 7). This evidence suggests that PCDH10 can induce apoptosis of pancreatic cancer cells. 


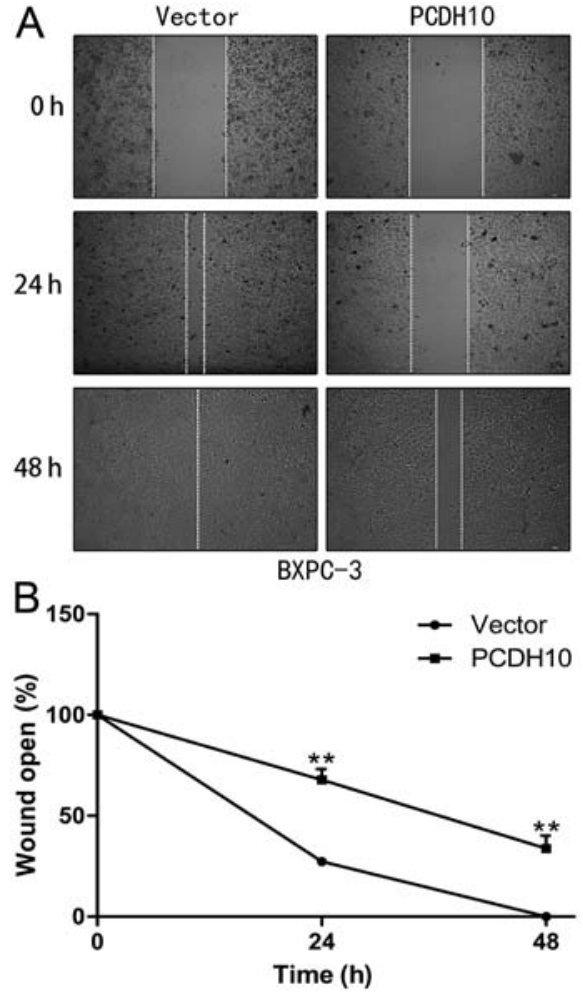

Figure 5. The effects of PCDH10 overexpression on cell migration ability of BxPC-3. (A) Representative images of wound healing assay. (B) The quantitative analysis of wound healing. ${ }^{* *} \mathrm{P}<0.01$ compared to the vector (inverted fluorescence microscope, $\mathrm{x} 100$ ).

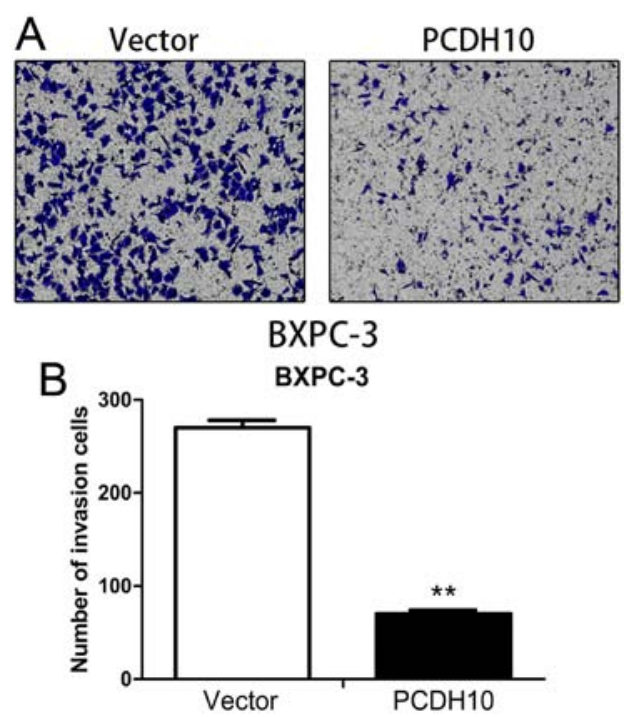

Figure 6. The effects of PCDH10 overexpression on cell invasion ability of BxPC-3. (A) The representative images of Transwell invasion assay. (B) The quantitative analysis of Transwell invasion assay. ${ }^{* *} \mathrm{P}<0.01$ compared to the vector (crystal violet staining, $\mathrm{x} 100$ ).

The effect of PCDH10 on the key cell apoptosis regulators. The flow cytometry results confirmed that PCDH10 can induce apoptosis in pancreatic cancer cells, thus, we performed western blot analysis to detect the level of apoptosis-related proteins. Fig. 4 shows the pro-apoptosis proteins (PARP, caspase-3 and caspase-9) were increased and anti-apoptosis protein (bcl-2) was decreased. In addi-
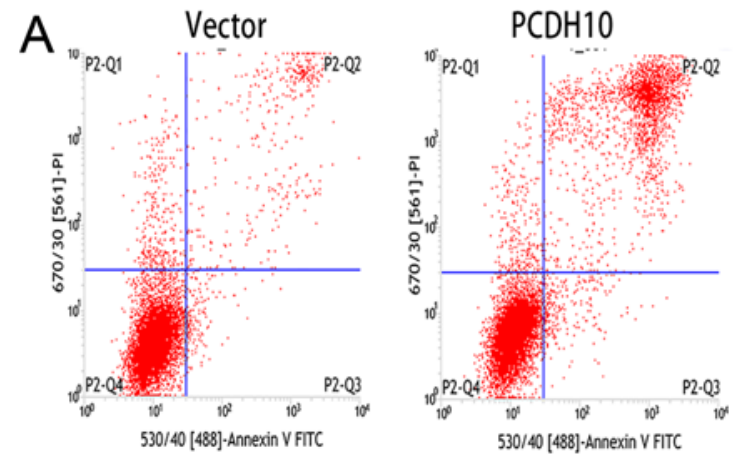

CAPAN-1

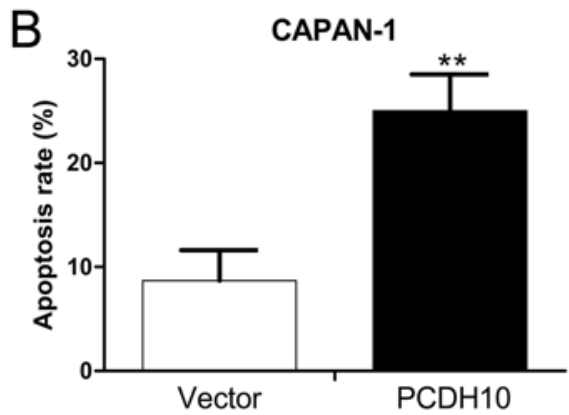

Figure 7. The effects of PCDH10 overexpression on cell apoptosis of Capan-1. (A) Representative images of cell apoptosis detected by combining flow cytometry and Annexin V-FITC/PI staining. (B) The quantitative analysis of cell apoptosis. ${ }^{*} \mathrm{P}<0.05$ compared to the vector.
A

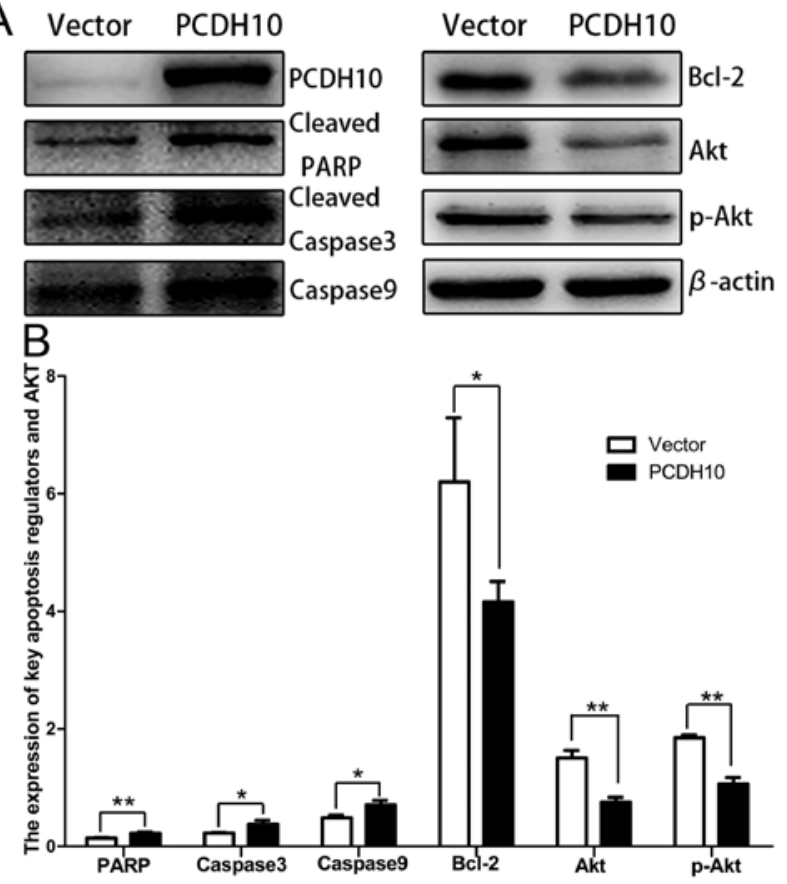

Figure 8. The effects of PCDH10 on the expression of the key apoptosis regulators and Akt proteins in Capan-1. (A) Representative images of western blot analysis. (B) (B) The quantitative analysis of the expression of PARP, caspase-3, caspase-9, Bcl-2, AKT and p-AKT; ${ }^{*} \mathrm{P}<0.05,{ }^{* *} \mathrm{P}<0.01$ compared to the vector, each sample was normalized to $\beta$-actin.

tion, we detected the AKT and p-AKT levels upstream of the apoptosis pathway, the proteins (AKT and p-AKT) were decreased $(\mathrm{P}<0.05$; Fig. 8). These results indicate that 
PCDH10 induced pancreatic cancer cell apoptosis may be via regulating the AKT pathway.

\section{Discussion}

The generation of most cancers involving pancreatic cancer are due to the genetic and epigenetic alterations of tumor suppressor genes (TSGs). Genetic alterations often accompany gene mutations which can change the DNA sequence. For instance, the tumor suppressor gene TP53 has frequent mutations resulting in inactivation in pancreatic cancer (18). Several studies have suggested that epigenetic alterations are important mechanism for tumor suppressor genes silencing, and aberrant DNA methylation could be a novel tumor marker for early diagnose and prognosis (19-21). DNA methylation as a tumor marker has some advantages: first, aberrant DNA methylation is more common as an early event in cancers; second, the techniques of detecting aberrant DNA methylation are simple, rapid and sensitive; finally, DNA is more stable than any other molecular markers similar to proteins. In DNA methylation, unlike genetic alteration, the silence of gene expression resulted by methylation alteration can be reversed by pharmacologic demethylation, this indicates that aberrant DNA methylation is a process recovering the expression of TSGs which changed by epigenetics and restoring their tumor suppression functions in cancer. Many TSGs and increasing number of genes with functional cancer suppression are silenced in pancreatic cancer by promoter methylation. Specifically, the inactivation of p16 tumor suppressor gene by promoter methylation in pancreatic cancer is well known (22). This indicated that abnormal methylation of TSGs contributes to pancreatic cancer pathogenesis.

In the present study, we explored PCDH10 in the expression, epigenetic alteration, biological function in pancreatic cancer cells. Our results demonstrate that the PCDH10 expression is downregulated in pancreatic cancer cells due to DNA promoter hypermethylation, which accords with the status of PCDH10 in other types of cancer, and this suggests that the aberrant DNA methylation of PCDH10 could be a new biomarker for detecting pancreatic cancer at an early stage. Re-expression of PCDH10 significantly suppresses cell proliferation and colony formation, markedly inhibits migration and invasion ability, and induces apoptosis in pancreatic cancer cells. These results suggest that PCDH10 have tumor suppressor function, which is consistent with previous studies on PCDH10 in gastric cancer and in colorectal cancer $(23,24)$. Thus, recovering PCDH10 expression and restoring its function shows promise as a new therapy for treating pancreatic cancer. Unfortunately, we did not collect enough pancreatic tissue samples, thus, we could not analyze the relationship between aberrant DNA methylation of PCDH10 and clinical features in pancreatic cancer.

Protocadherins play an important role in signal transduction, implying that PCDH10 action as tumor suppressor gene may be via mediating cancer-related signaling pathway. For example, PCDH10 inhibits the proliferation of multiple myeloma cells via downregulation of Wnt/B-catenin/BCL-9 signaling pathway (25), and induces apoptosis of multiple myeloma cells by inhibiting the NF- $\kappa B$ pathway (26). In this study, we explored the apoptosis mechanisms of PCDH10 in pancreatic cancer cells. The results showed that PCDH10 may regulate the AKT pathway to induce the apoptosis of pancreatic cancer cells.

Apoptosis is a regulated cellular suicide mechanism characterized by nuclear fragmentation, membrane blebbing and formation of apoptotic bodies (27). Apoptosis pathway has been classified into mitochondria-mediated pathway and receptor-mediated pathway (28). The natural death mechanism can lead to several diseases involving cancer when it is abnormally regulated. PARP, poly(ADP-ribose) polymerase, appears to be involved in DNA repair in response to environmental stress. It was reported to have a key role in caspase-independent cell death pathway defined as necroptosis (29). Caspases, a family of cysteine proteases, is divided into initiator and effector caspases. Initiator caspases such as caspase-9, form apoptosome complex and activate downstream effector caspases by binding large adaptor molecules which promote caspase activation. Caspase-3, a member of effector caspases, is the primary executioner of programmed cell death, as it is directly or indirectly responsible for cleavage of many proteins and initiator caspases involved in apoptosis. Bcl-2 family of proteins is another important regulator of apoptosis, it is divided into anti-apoptosis proteins and proapoptosis proteins, according to the domain and function. Bcl-2 belongs to anti-apoptosis proteins, locating mainly on the outer mitochondria membrane. They protect the cells by interacting with mitochondria protein, preventing damage to the membrane, and they can inhibit the pro-apoptosis factors released $(28,30)$.

PI3K/AKT signaling pathway has become a major focus of attention for its critical role in regulating some of the most fundamental aspects of cell growth, proliferation, survival, transcription, and protein synthesis, disorder of the PI3K/AKT pathway is contributing to many diseases including cancer, diabetes, cardiovascular and neurological diseases (31). In addition, AKT pathway is the commonly activated signaling pathway in most cancers. Serine/threonine kinase Akt, the key protein of PI3K/AKT signaling pathway, is the major mediator of cell apoptosis by directly inhibiting pro-apoptosis proteins like Bad, caspase-9 and pro-apoptosis signals generated by transcription factors such as FoxO1 to regulate cell apoptosis (32).

\section{References}

1. Jemal A, Siegel R, Ward E, Hao Y, Xu J, Murray T and Thun MJ: Cancer statistics, 2008. CA Cancer J Clin 58: 71-96, 2008.

2. Hong SM, Park JY, Hruban RH and Goggins M: Molecular signatures of pancreatic cancer. Arch Pathol Lab Med 135: 716-727, 2011.

3. Wolffe AP and Matzke MA: Epigenetics: Regulation through repression. Science 286: 481-486, 1999.

4. Singal R and Ginder GD: DNA methylation. Blood 93: 4059-4070, 1999.

5. Esteller M: Cancer epigenomics: DNA methylomes and histonemodification maps. Nat Rev Genet 8: 286-298, 2007.

6. Merlo A, Herman JG, Mao L, Lee DJ, Gabrielson E, Burger PC, Baylin SB and Sidransky D: 5' CpG island methylation is associated with transcriptional silencing of the tumour suppressor p16/ CDKN2/MTS1 in human cancers. Nat Med 1: 686-692, 1995.

7. Xiang T, Li L, Yin X, Yuan C, Tan C, Su X, Xiong L, Putti TC, Oberst M, Kelly K, et al: The ubiquitin peptidase UCHL1 induces G0/G1 cell cycle arrest and apoptosis through stabilizing p53 and is frequently silenced in breast cancer. PLoS One 7: e29783, 2012. 
8. Li L, Ying J, Tong X, Zhong L, Su X, Xiang T, Shu X, Rong R, Xiong L, Li H, et al: Epigenetic identification of receptor tyrosine kinase-like orphan receptor 2 as a functional tumor suppressor inhibiting $\beta$-catenin and AKT signaling but frequently methylated in common carcinomas. Cell Mol Life Sci 71: 2179-2192, 2014.

9. Redies C, Vanhalst K and Roy F: delta-Protocadherins: Unique structures and functions. Cell Mol Life Sci 62: 2840-2852, 2005.

10. Morishita $\mathrm{H}$ and Yagi T: Protocadherin family: Diversity, structure, and function. Curr Opin Cell Biol 19: 584-592, 2007.

11. Yu JS, Koujak S, Nagase S, Li CM, Su T, Wang X, Keniry M, Memeo L, Rojtman A, Mansukhani M, et al: PCDH8, the human homolog of PAPC, is a candidate tumor suppressor of breast cancer. Oncogene 27: 4657-4665, 2008.

12. Hu X, Sui X, Li L, Huang X, Rong R, Su X, Shi Q, Mo L, Shu X, Kuang Y, et al: Protocadherin 17 acts as a tumour suppressor inducing tumour cell apoptosis and autophagy, and is frequently methylated in gastric and colorectal cancers. J Pathol 229: 62-73, 2013.

13. Lv J, Zhu P, Yang Z, Li M, Zhang X, Cheng J, Chen X and Lu F: $\mathrm{PCDH} 20$ functions as a tumour-suppressor gene through antagonizing the $\mathrm{Wnt} / \beta$-catenin signalling pathway in hepatocellular carcinoma. J Viral Hepat 22: 201-211, 2015.

14. Li Z, Chim JC, Yang M, Ye J, Wong BC and Qiao L: Role of PCDH10 and its hypermethylation in human gastric cancer. Biochim Biophys Acta 1823: 298-305, 2012.

15. Zhong X, Zhu Y, Mao J, Zhang J and Zheng S: Frequent epigenetic silencing of PCDH10 by methylation in human colorectal cancer. J Cancer Res Clin Oncol 139: 485-490, 2013.

16. Tang X, Yin X, Xiang T, Li H, Li F, Chen L and Ren G: Protocadherin 10 is frequently downregulated by promoter methylation and functions as a tumor suppressor gene in non-small cell lung cancer. Cancer Biomark 12: 11-19, 2012-2013.

17. Ying J, Li H, Seng TJ, Langford C, Srivastava G, Tsao SW, Putti T, Murray P, Chan AT and Tao Q: Functional epigenetics identifies a protocadherin PCDH10 as a candidate tumor suppressor for nasopharyngeal, esophageal and multiple other carcinomas with frequent methylation. Oncogene 25: 1070-1080, 2006.

18. Redston MS, Caldas C, Seymour AB, Hruban RH, da Costa L, Yeo CJ and Kern SE: p53 mutations in pancreatic carcinoma and evidence of common involvement of homocopolymer tracts in DNA microdeletions. Cancer Res 54: 3025-3033, 1994.

19. Yi JM, Guzzetta AA, Bailey VJ, Downing SR, Van Neste L, Chiappinelli KB, Keeley BP, Stark A, Herrera A, Wolfgang C, et al: Novel methylation biomarker panel for the early detection of pancreatic cancer. Clin Cancer Res 19: 6544-6555, 2013
20. Roperch JP, Incitti R, Forbin S, Bard F, Mansour H, Mesli F, Baumgaertner I, Brunetti F and Sobhani I: Aberrant methylation of NPY, PENK, and WIF1 as a promising marker for blood-based diagnosis of colorectal cancer. BMC Cancer 13: 566, 2013.

21. Balgkouranidou I, Matthaios D, Karayiannakis A, Bolanaki H, Michailidis P, Xenidis N, Amarantidis K, Chelis L, Trypsianis G, Chatzaki E, et al: Prognostic role of APC and RASSF1A promoter methylation status in cell free circulating DNA of operable gastric cancer patients. Mutat Res 778: 46-51, 2015.

22. Schutte M, Hruban RH, Geradts J, Maynard R, Hilgers W, Rabindran SK, Moskaluk CA, Hahn SA, Schwarte-Waldhoff I, Schmiegel W, et al: Abrogation of the Rb/p16 tumor-suppressive pathway in virtually all pancreatic carcinomas. Cancer Res 57: 3126-3130, 1997.

23. Yu J, Cheng YY, Tao Q, Cheung KF, Lam CN, Geng H, Tian LW, Wong YP, Tong JH, Ying JM, et al: Methylation of protocadherin 10 , a novel tumor suppressor, is associated with poor prognosis in patients with gastric cancer. Gastroenterology 136: 640-51.e1, 2009.

24. Jao TM, Tsai MH, Lio HY, Weng WT, Chen CC, Tzeng ST, Chang CY, Lai YC, Yen SJ, Yu SL, et al: Protocadherin 10 suppresses tumorigenesis and metastasis in colorectal cancer and its genetic loss predicts adverse prognosis. Int J Cancer 135: 2593-2603, 2014.

25. Xu Y, Yang Z, Yuan H, Li Z, Li Y, Liu Q and Chen J: PCDH10 inhibits cell proliferation of multiple myeloma via the negative regulation of the Wnt/ $\beta$-catenin/BCL-9 signaling pathway. Oncol Rep 34: 747-754, 2015

26. Li Z, Yang Z, Peng X, Li Y, Liu Q and Chen J: Nuclear factor- $\kappa B$ is involved in the protocadherin-10-mediated pro-apoptotic effect in multiple myeloma. Mol Med Rep 10: 832-838, 2014.

27. Degterev A and Yuan J: Expansion and evolution of cell death programmes. Nat Rev Mol Cell Biol 9: 378-390, 2008.

28. Indran IR, Tufo G, Pervaiz $S$ and Brenner $C$ : Recent advances in apoptosis, mitochondria and drug resistance in cancer cells. Biochim Biophys Acta 1807: 735-745, 2011.

29. Drel' VR, Shymans'kyı̆ IO, Sybirna NO andVelykyı̆ MM: Role of PARP and protein poly-ADP-ribosylation process in regulation of cell functions. Ukr Biokhim Zh 83: 5-34, 2011.

30. Fuchs Y and Steller H: Programmed cell death in animal development and disease. Cell 147: 742-758, 2011.

31. Hers I, Vincent EE and Tavaré JM: Akt signalling in health and disease. Cell Signal 23: 1515-1527, 2011.

32. Manning BD and Cantley LC: AKT/PKB signaling: Navigating downstream. Cell 129: 1261-1274, 2007. 